\title{
Curcumin exerts antitumor effects in retinoblastoma cells by regulating the JNK and p38 MAPK pathways
}

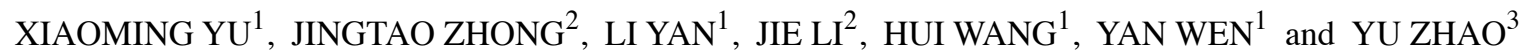 \\ ${ }^{1}$ Department of Ophthalmology, Shandong Jiaotong Hospital, Jinan, Shandong 250031; ${ }^{2}$ Department of General Surgery, \\ Qianfoshan Hospital, Shandong University, Jinan, Shandong 250014; ${ }^{3}$ Department of Otolaryngology, \\ Shandong Jiaotong Hospital, Jinan, Shandong 250031, P.R. China
}

Received October 23, 2015; Accepted July 7, 2016

DOI: $10.3892 /$ ijmm.2016.2676

\begin{abstract}
Curcumin, a naturally occurring polyphenolic compound present in turmeric (Curcuma longa), exerts antitumor effects in various types of malignancy. However, the precise mechanisms responsible for the effects of curcumin on retinoblastoma (RB) cells have not been fully explored. In the present study, the molecular mechanisms by which curcumin exerts its anticancer effects in RB Y79 cells were investigated. The results showed that curcumin reduced cell viability in Y79 cells. Curcumin induced G1 phase arrest through downregulating the expression of cyclin D3 and cyclin-dependent kinase (CDK)2/6 and upregulating the expression of CDK inhibitor proteins $\mathrm{p} 21$ and p27. Curcumin-induced apoptosis of Y79 cells occurred through the activation of caspases-9/-3. Moreover, flow cytometric analysis showed that curcumin induced mitochondrial membrane potential $(\Delta \Psi \mathrm{m})$ collapse in Y79 cells. We also found that curcumin induced the phosphorylation of c-Jun $\mathrm{N}$-terminal kinase (JNK) and p38 mitogen-activated protein kinase (MAPK). JNK and p38 MAPK inhibitors significantly suppressed curcumin-induced activation of caspases-9/-3 and inhibited the apoptosis of Y79 cells. Taken together, our results suggest that curcumin induced the apoptosis of Y79 cells through the activation of JNK and p38 MAPK pathways. These findings provide a novel treatment strategy for human RB.
\end{abstract}

\section{Introduction}

Retinoblastoma $(\mathrm{RB})$ is the most common intraocular malignancy in children with an incidence of 1 in 15,000 to 1 in 20,000 births, accounting for approximately $4 \%$ of all pediatric malignancies $(1,2)$. Most cases of unilateral RB are caused by sporadic somatic mutations in the RB1 gene, representing approximately $60 \%$ of all RB cases whereas about $40 \%$ of cases occur in infants

Correspondence to: Dr Xiaoming Yu, Department of Ophthalmology, Shandong Jiaotong Hospital, 12 Wuyingshan Middle Road, Jinan, Shandong 250031, P.R. China

E-mail: yuxm1987@163.com

Key words: retinoblastoma, curcumin, apoptosis, cell cycle, c-Jun $\mathrm{N}$-terminal kinase, p38 mitogen-activated protein kinase with germline mutations (3). Treatment strategies for RB include intravenous chemoreduction, enucleation, transpupillary thermotherapy, cryotherapy, thermotherapy, laser photocoagulation, brachytherapy, plaque radiotherapy, orbital exenteration, external beam radiotherapy, and chemotherapy, depending on the stage of tumor development and the location and size of the primary tumor $(4,5)$. Despite progress in the treatment of $\mathrm{RB}$, a number of these treatments have possible side effects, such as blindness, infection, fever, gastrointestinal toxicity and neurotoxicity (6). Therefore, there is an urgent need for the development of novel therapeutic agents for use in the management of RB.

Curcumin [also known as diferuloylmethane and 1,7-bis(4-hydroxy 3-methoxyphenyl)-1,6-heptadiene-3,5-dione], is a naturally occurring polyphenolic compound present in turmeric (Curcuma longa) which has been employed to treat a number of diseases including asthma, bronchial hyperactivity, allergy, anorexia, coryza, cough, sinusitis and hepatic disease in Asian countries for thousands of years (7). Recently, accumulating evidence has demonstrated that curcumin exerts antitumor effects on various types of cancer cells as well as being non-cytotoxic to normal cells (8-13). However, the precise mechanisms responsible for the effects of curcumin on RB cells have not been fully explored. Several studies have reported that curcumin exerts antitumor effects through inducing apoptosis in a variety of types of cancer cells which involves the activation of caspases (14), mitochondrial dysfunction triggered by enhanced Bax levels (15), and pro-apoptotic endoplasmic reticulum stress (16). Furthermore, a number of studies have revealed that curcumin exerts antitumor effects through mediating various cellular signaling pathways including nuclear factor $\kappa \mathrm{B}(\mathrm{NF}-\kappa \mathrm{B})(17)$, signal transducer and activator of transcription 3 (STAT3) (18), protein kinase B (PKB/Akt) (19), mitogenactivated protein kinase (MAPK) (20), and other pathways. In this study, we examined the molecular mechanism responsible for curcumin-induced cytotoxicity in human RB cells.

\section{Materials and methods}

Cell culture. The human RB cell line Y79 was obtained from the American Type Culture Collection (ATCC; Manassas, VA, USA). The cells were cultured in RPMI-1640 medium containing $10 \%$ fetal bovine serum, $1 \%$ penicillin, and streptomycin (Gibco, Grand Island, $\mathrm{NY}, \mathrm{USA}$ ) at $37^{\circ} \mathrm{C}$ in $95 \%$ air and $5 \% \mathrm{CO}_{2}$. 
Reagents and antibodies. Curcumin and ZVAD-FMK were purchased from Sigma-Aldrich (San Diego, CA, USA). SP600125 was obtained from AG Scientific, Inc. (San Diego, CA, USA). SB203580 was purchased from Calbiochem (San Diego, CA, USA). Antibodies against cyclin D3 (ab63535), p21 (ab109520), p27 KIP1 (ab32034), cytochrome $c$ (ab53056), caspase-3 (ab90437), caspase-9 (ab25758), and GAPDH (ab37168) were purchased from Abcam (Cambridge, UK). Antibodies against cyclin-dependent kinase (CDK)2 (\#2546), CDK6 (\#13331), c-Jun N-terminal kinase (JNK; \#9252), p38 MAPK (\#9212), phospho-JNK (Thr183/Tyr185; \#9251), and phospho-p38 MAPK (Thr180/Tyr182; \#9211) were obtained from Cell Signaling Technology (Danvers, MA, USA).

Cell treatment. Curcumin was dissolved in dimethylsulfoxide (DMSO; Sigma-Aldrich, St. Louis, MO, USA) and diluted immediately prior to each experiment. Final curcumin concentrations of $10-80 \mu \mathrm{M}$ were obtained by dilution in culture media. Controls containing $0.07 \%$ DMSO were included in all experiments. In order to inhibit caspase activity, Y79 cells were treated with ZVAD-FMK at a concentration of $50 \mu \mathrm{M}$ for $1 \mathrm{~h}$ prior to curcumin treatment. Y79 cells were treated in the absence or presence of $20 \mu \mathrm{M} \mathrm{JNK}$ inhibitor (SP600125) or p38 MAPK inhibitor (SB203580) for $1 \mathrm{~h}$, then treated with $80 \mu \mathrm{M}$ curcumin for $24 \mathrm{~h}$ before examining the phosphorylation levels of JNK and p38 MAPK.

Measurement of cell viability. The Cell Counting Kit-8 (CCK-8) (Dojindo Molecular Technologies, Inc., Beijing, China) was used to determine cell viability. Briefly, cells $\left(5 \times 10^{3} /\right.$ well) were incubated with curcumin-containing RPMI-1640 in 96-well plates for $24 \mathrm{~h}$, and then the culture medium was replaced with fresh medium containing $10 \mathrm{ml} \mathrm{CCK-8} \mathrm{solu-}$ tion. The cells were further incubated for $2 \mathrm{~h}$ at $37^{\circ} \mathrm{C}$, and the optical density (OD) at $450 \mathrm{~nm}$ was measured.

Cell cycle analysis by flow cytometry. Y79 cells were seeded at a density of $4 \times 10^{5}$ in 6-well culture plates, grown overnight in medium containing $10 \%$ FBS, and treated with or without various concentrations of curcumin $(0-80 \mu \mathrm{M})$ for $24 \mathrm{~h}$. Cell cycle analysis was then performed. Briefly, the cells were suspended in $0.5 \mathrm{ml}$ propidium iodide (PI) solution, and incubated for $30 \mathrm{~min}$ in the dark according to the manufacturer's instructions. The cell cycle distribution was analyzed by flow cytometry [fluorescence-activated cell sorting (FACS) analysis; BD Biosciences, San Jose, CA, USA].

Analysis of apoptosis by flow cytometry. Apoptosis was examined using the Annexin V-fluorescein isothiocyanate (FITC) Apoptosis Detection kit (BioVision Inc., Mountain View, CA, USA), according to the manufacturer's instructions.

Western blot analysis. Protein concentrations in the cell extracts were determined (Bio-Rad, Richmond, CA, USA). Briefly, equal amounts of each sample were resolved in SDS-PAGE gels and then transferred to a polyvinylidene fluoride (PVDF) membrane (Millipore, Billerica, MA, USA), and probed with the primary antibodies described above in Reagents and antibodies. Protein band intensities were quanti-

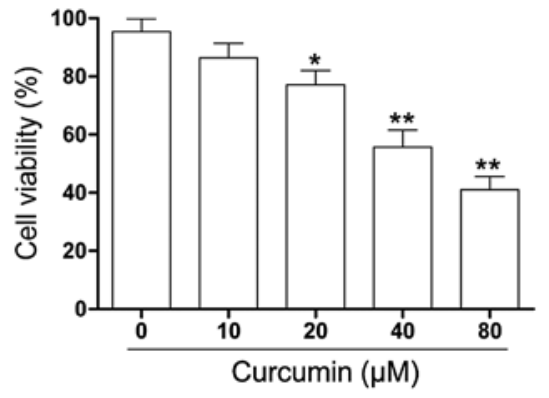

Figure 1. Effect of curcumin on the viability of human retinoblastoma (RB) Y79 cells in vitro. Cell viability was assessed using the Cell Counting Kit-8 (CCK-8) assay. Y79 cells were treated with various concentrations $(0-80 \mu \mathrm{M})$ of curcumin for $24 \mathrm{~h}$. Data are expressed as the percentage of control cells and are the means \pm SD of three separate experiments. ${ }^{*} \mathrm{P}<0.05,{ }^{* *} \mathrm{P}<0.01$ vs. control.

fied by densitometric analysis using ImageJ software (National Institutes of Health, Bethesda, MD, USA).

Determination of mitochondrial membrane potential $(\Delta \Psi \mathrm{m})$. Briefly, Y79 cells were exposed to various concentrations of curcumin $(0-80 \mu \mathrm{M})$ for $24 \mathrm{~h}$. The cells were then harvested and incubated with $40 \mathrm{nmol} / 1 \mathrm{DiOC}_{6}(\mathrm{Abcam})$ at $37^{\circ} \mathrm{C}$ in the dark for $20 \mathrm{~min}$. Finally, the mean fluorescence intensity (MFI) was determined by peforming flow cytometric analysis.

Statistical analysis. The data are expressed as the means \pm standard deviation (SD). Comparisons were made using a one-way ANOVA followed by Dunnett's test with SPSS software (version 17.0; SPSS Inc., Chicago, IL, USA). P $<0.05$ was considered to indicate a statistically significant difference.

\section{Results}

Curcumin significantly inhibits cell viability in Y79 cells. To explore the effect of curcumin on the cell viability of RB cells in vitro, human RB (Y79) cells were treated with varying concentrations of curcumin $(0-80 \mu \mathrm{M})$ for $24 \mathrm{~h}$ and changes in cell viability were assessed by the CCK- 8 assay. As shown in Fig. 1, the viability of the Y79 cells exposed to curcumin was significantly lower compared with that of the control cells. A sharp decrease in cell viability was present at a curcumin concentration of $40 \mu \mathrm{M}$.

Curcumin induces cell cycle arrest of Y79 cells. We explored whether curcumin induces cell cycle arrest in human RB cells. The Y79 cells were exposed to various concentrations of curcumin for $24 \mathrm{~h}$ and then analyzed for alterations in the cell cycle by flow cytometry. As shown in Fig. 2A, curcumin-treated Y79 cells were inhibited in the G1 phase after $24 \mathrm{~h}$ of treatment. To further examine the molecular mechanisms underlying curcumin-induced G1 phase arrest, the cells were treated with various concentrations of curcumin $(0-80 \mu \mathrm{M})$ for $24 \mathrm{~h}$, and harvested for protein extraction and western blot analysis. As shown in Fig. 2B and C, the protein expression of cyclin D3, $\mathrm{CDK} 2$ and CDK6 was markedly reduced in the curcumin-treated Y79 cells. Moreover, the levels of the CDK inhibitor proteins p21 and p27 were significantly upregulated following the exposure of Y79 cells to curcumin. These results suggest that 
A

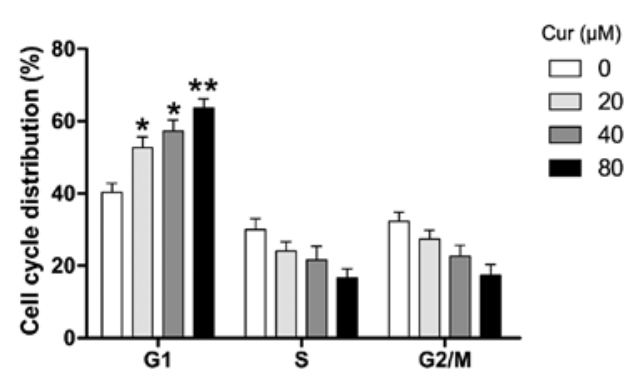

C

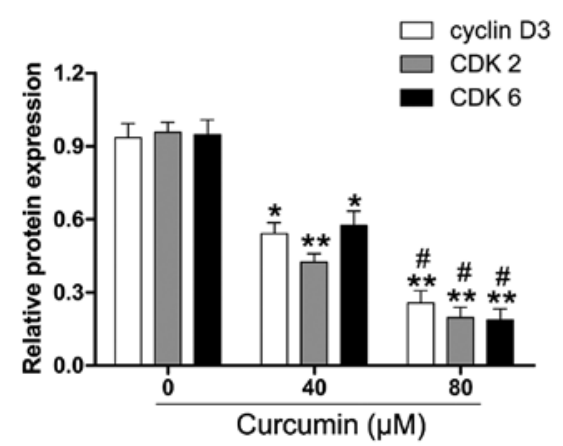

B
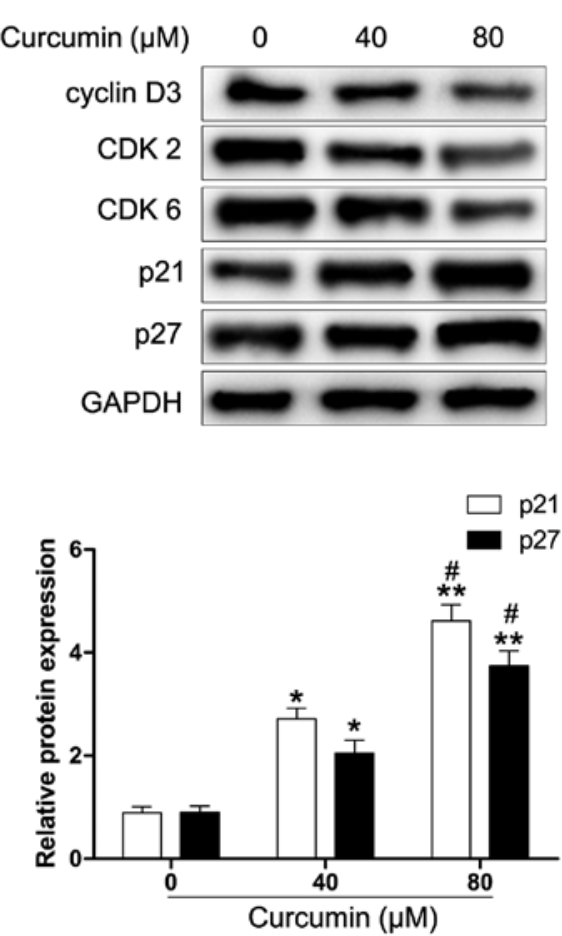

Figure 2. Curcumin induces cell cycle arrest in Y79 cells in vitro. (A) Cell cycle distribution of Y79 cells was determined $24 \mathrm{~h}$ after treatment with various concentrations of curcumin $(0-80 \mu \mathrm{M})$. Data are expressed as the means \pm SD of three independents experiments. ${ }^{*} \mathrm{P}<0.05$, ${ }^{* *} \mathrm{P}<0.01 \mathrm{vs}$. control. (B and C) Cells were treated with curcumin $(0-80 \mu \mathrm{M})$. The cell lysates were harvested at $24 \mathrm{~h}$ and evaluated by western blot analysis using specific antibodies against cyclin D3, cyclin-dependent kinase (CDK)2, CDK6, p21, and p27. Levels of GAPDH served as a loading control. The results shown are representative of at least three independent experiments. ${ }^{*} \mathrm{P}<0.05,{ }^{* * *} \mathrm{P}<0.01$ vs. control. ${ }^{*} \mathrm{P}<0.05$ vs. curcumin $(40 \mu \mathrm{M})$ group.

A

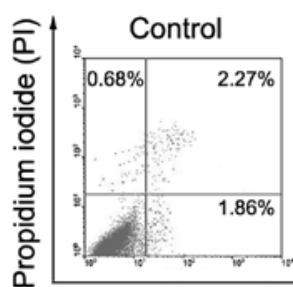

B

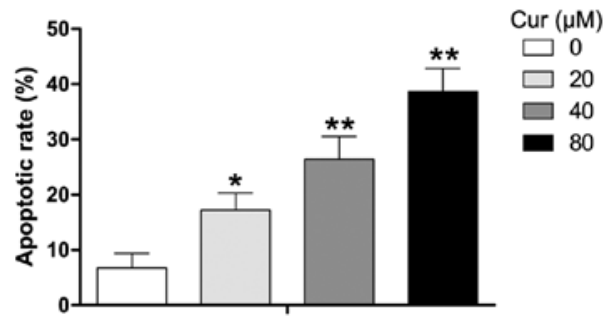

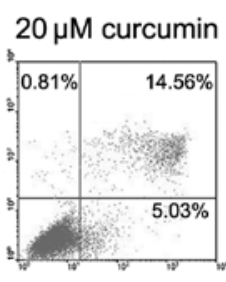
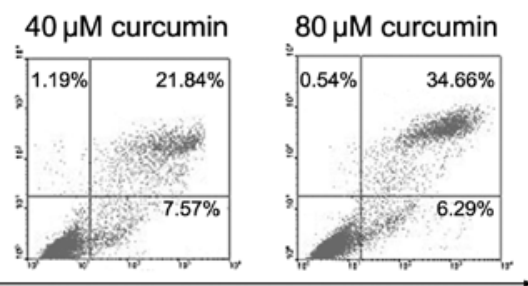

Annexin V-FITC

C

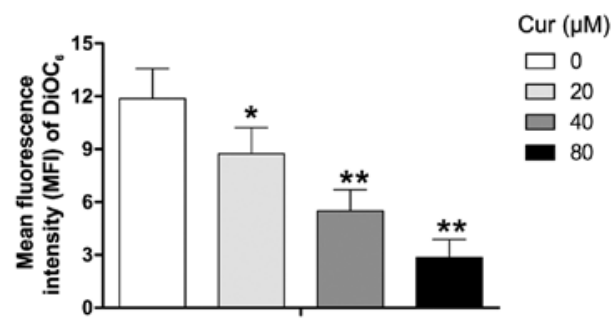

Figure 3. Curcumin induces the apoptosis of Y79 cells in vitro. (A) Y79 cells were untreated (control) or exposed to curcumin (20-80 $\mu \mathrm{M})$ for $24 \mathrm{~h}$. Apoptotic cells were analyzed by flow cytometry (FACS analysis) with propidium iodide (PI) and Annexin V-fluorescein isothiocyanate (FITC) staining. (B) Quantitative analysis of the total apoptotic (early and late) population. Data are presented as the means $\pm \mathrm{SD}$ of three independents experiments. ${ }^{*} \mathrm{P}<0.05$, ${ }^{* *} \mathrm{P}<0.01$ vs. control. (C) Curcumin induced $\Delta \Psi \mathrm{m}$ collapse in Y79 cells. Y79 cells were untreated (control) or treated with curcumin (20-80 $\mu \mathrm{M})$ for $24 \mathrm{~h}$. The mean fluorescence intensity (MFI) of $\mathrm{DiOC}_{6}$ was obtained by flow cytometric analysis. Data are presented as the means $\pm \mathrm{SD}$ of three independents experiments. ${ }^{*} \mathrm{P}<0.05,{ }^{* *} \mathrm{P}<0.01$ vs. control. Cur, curcumin.

curcumin-induced G1 phase cell cycle arrest in human RB cells may be regulated through the cyclin-CDK checkpoint.

Curcumin induces the apoptosis of Y79 cells. To determine whether the effect of curcumin on cell viability was caused by apoptotic cell death, Y79 cells were exposed to various concentrations of curcumin $(0-80 \mu \mathrm{M})$ for $24 \mathrm{~h}$, and the extent of apoptosis was evaulated using the Annexin V/PI assay. As shown in Fig. 3A and B, the percentage of apoptotic cells (PI-negative/Annexin V-positive and 
A

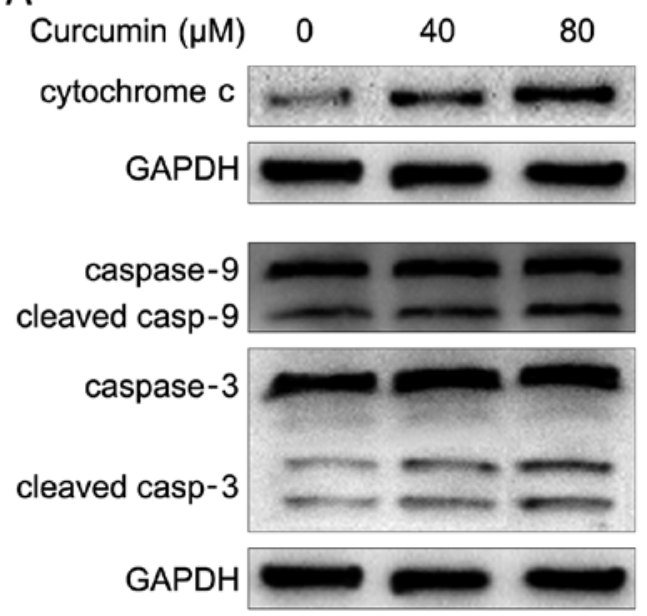

C

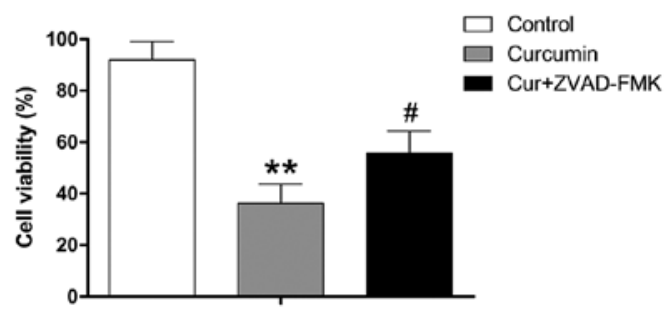

B

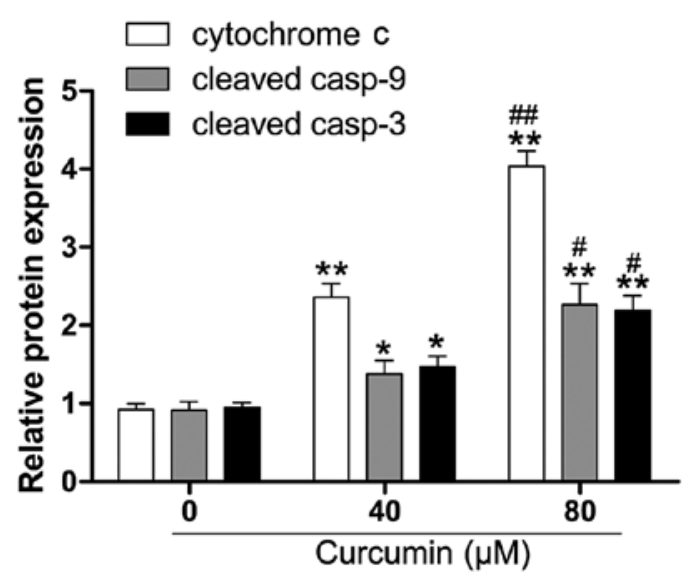

D

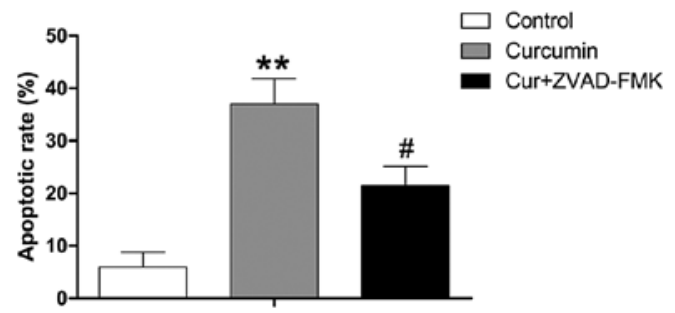

Figure 4. Curcumin induces the apoptosis of Y79 cells through intrinsic pathways. (A and B) Cells were treated with curcumin (0-80 $\mu \mathrm{M})$. The cell lysates were harvested at $24 \mathrm{~h}$ and evaluated by western blot analysis with specific antibodies against cytochrome $c$, caspase-9 and caspase-3. Levels of GAPDH served as a loading control. The results shown are representative of at least three independent experiments. ${ }^{*} \mathrm{P}<0.05,{ }^{* *} \mathrm{P}<0.01$ vs. control. ${ }^{\#} \mathrm{P}<0.05,{ }^{\# \#} \mathrm{P}<0.01$ vs. curcumin $(40 \mu \mathrm{M})$ group. (C) Cells were treated with $80 \mu \mathrm{M}$ curcumin for $24 \mathrm{~h}$ in the presence or absence of $50 \mu \mathrm{M}$ ZVAD-FMK. Cell viability was determined by the Cell Counting Kit- 8 (CCK-8) assay. Data are expressed as the percentage of control cells and are the means \pm SD of three separate experiments. ${ }^{* *} \mathrm{P}<0.01$ vs. control. ${ }^{\text {}} \mathrm{P}<0.05$ vs. curcumin group. (D) Curcumin-induced apoptosis was inhibited by $50 \mu \mathrm{M} Z \mathrm{ZVAD}-\mathrm{FMK}$ treatment. Data are presented as the means $\pm \mathrm{SD}$ of three independents experiments. ${ }^{* *} \mathrm{P}<0.01$ vs. control. ${ }^{\#} \mathrm{P}<0.05$ vs. curcumin group. Cur, curcumin.

PI-positive/Annexin V-positive) increased from 4.13 to 19.59 29.41 , and $40.95 \%$, respectively, after the Y79 cells were either untreated (control) or treated with 20,40 , and $80 \mu \mathrm{M}$ curcumin. DiOC $_{6}$, a lipophilic cationic dye, has been reported to specifically accumulate in the mitochondrial matrix depending on the $\Delta \Psi \mathrm{m}$ which is decreased in apoptotic cells $(21,22)$. To further ascertain the effects of curcumin on $\Delta \Psi \mathrm{m}$ in human RB cells, Y79 cells were exposed to various concentrations of curcumin $(0-80 \mu \mathrm{M})$ for $24 \mathrm{~h}$, and then analyzed in order to determine the MFI of $\mathrm{DiOC}_{6}$ by flow cytometry. We found that treatment with curcumin significantly decreased the MFI of $\mathrm{DiOC}_{6}$ (Fig. 3C). The data suggest that curcumin-induced apoptosis may occur through $\Delta \Psi \mathrm{m}$ dissipation in $\mathrm{Y} 79$ cells.

Curcumin induces the apoptosis of Y79 cells through intrinsic pathways. Accumulating evidence has revealed that $\Delta \Psi$ m plays an important role in regulating cellular functions. Disturbances of $\Delta \Psi \mathrm{m}$ may change the membrane dynamics of mitochondria and result in the release of cytochrome $c$, which triggers the formation of the apoptosome complex, and the subsequent activation of caspase-9. To determine whether curcumin induces apoptosis through the release of cytochrome $c$ and the activation of caspase-9 in human RB, Y79 cells were exposed to various concentrations of curcumin $(0-80 \mu \mathrm{M})$ for $24 \mathrm{~h}$. As shown in Fig. 4A and B, curcumin significantly enhanced cytochrome $c$ levels in a dose-dependent manner. We also observed that the released cytochrome $c$ triggered the activation of caspase-9 and caspase-3 (Fig. 4B). Moreover, we used the pan-caspase inhibitor (ZVAD-FMK) to evaluate the effect of curcumin on apoptotic cell death in Y79 cells. As depicted in Fig. 4C, pre-treatment with ZVAD-FMK attenuated the curcumin-induced reduction of viability in the Y79 cells. We also found that ZVAD-FMK attenuated the apoptotic effect of curcumin in the Y79 cells, which suggested that the activation of caspases is involved in curcumin-regulated apoptosis of human RB cells (Fig. 4D).

$J N K$ and p38 MAPK signaling play essential roles in caspase-9/-3 activation induced by curcumin. Previous studies have demonstrated that JNK and $\mathrm{p} 38$ MAPK are involved in the effects exerted by curcumin in tumor cells $(10,23)$. However, the role of MAPKs in curcumin-induced apoptosis of RB cells was not examined. In this study, we explored whether JNK and p38 MAPK were activated in curcumin-treated Y79 cells. As shown in Fig. 5, curcumin induced an increase in the phosphorylation of JNK and p38 MAPK in the Y79 cells. To determine whether JNK and p38 MAPK were necessary for curcumin-induced apoptosis, we examined the relationships among caspases-9/-3 and JNK, and p38 MAPK in the presence of curcumin. The Y79 cells were treated in the absence 
A
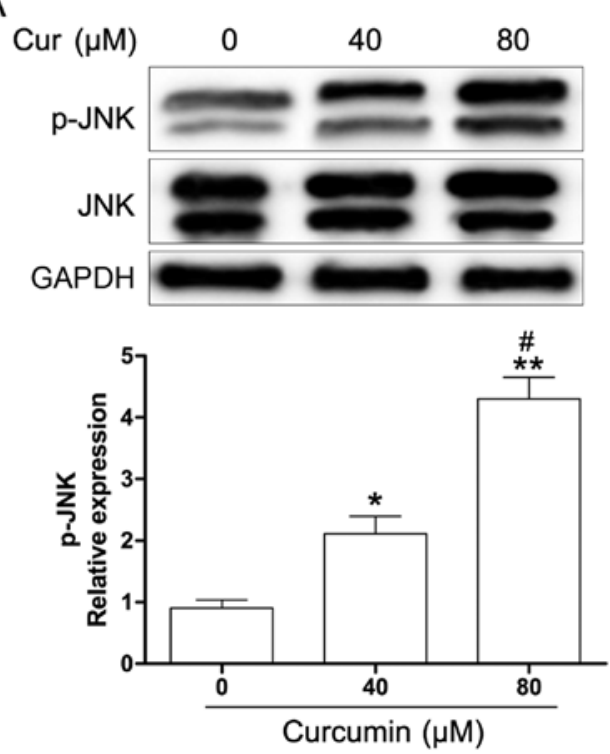

B
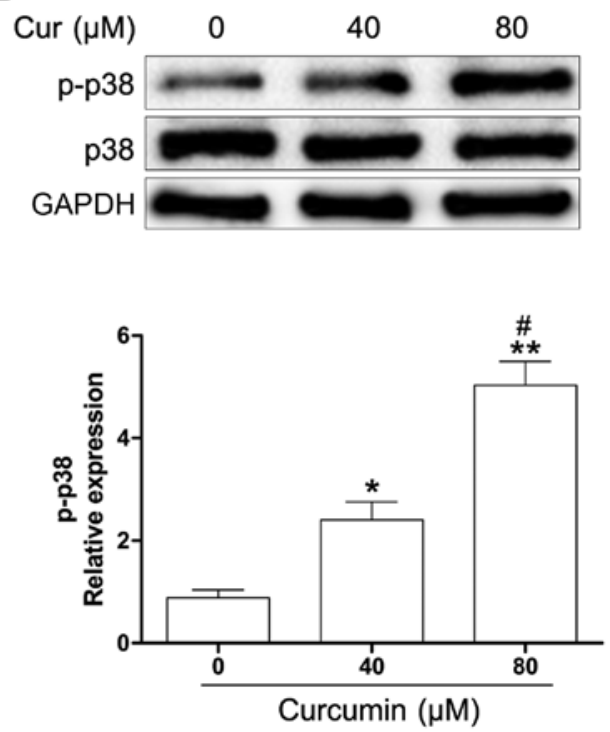

Figure 5. Curcumin induces c-Jun N-terminal kinase (JNK) and p38 mitogen-activated protein kinase (MAPK) phosphorylation in Y79 cells. (A and B) Y79 cells were incubated with various concentrations of curcumin $(0-80 \mu \mathrm{M})$ for $24 \mathrm{~h}$. The levels of both total and phosphorylated (p-)JNK and p38 MAPK were evaluated by western blot analysis. Levels of GAPDH served as a loading control. The results shown are representative of at least three independent experiments. ${ }^{*} \mathrm{P}<0.05,{ }^{* *} \mathrm{P}<0.01$ vs. control. ${ }^{\#} \mathrm{P}<0.05$ vs. curcumin $(40 \mu \mathrm{M})$ group. Cur, curcumin.

A

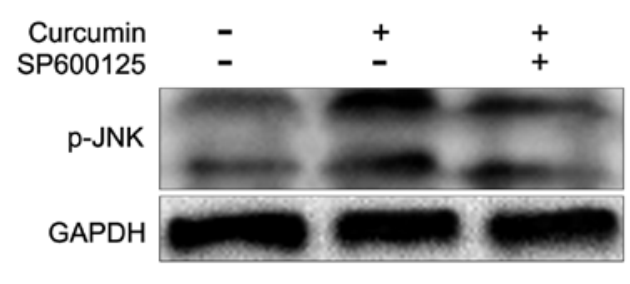

B

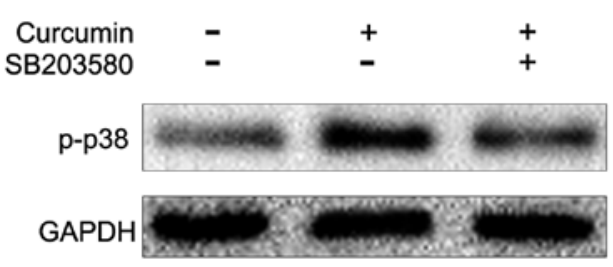

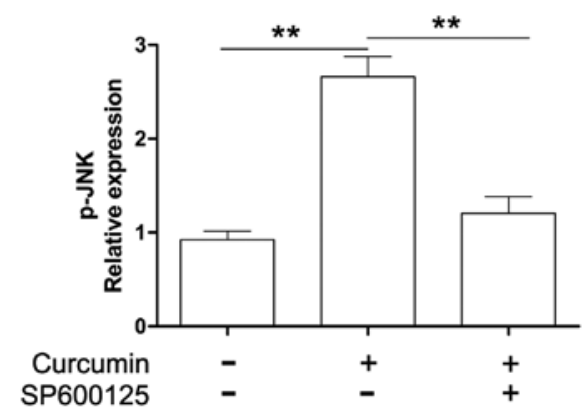

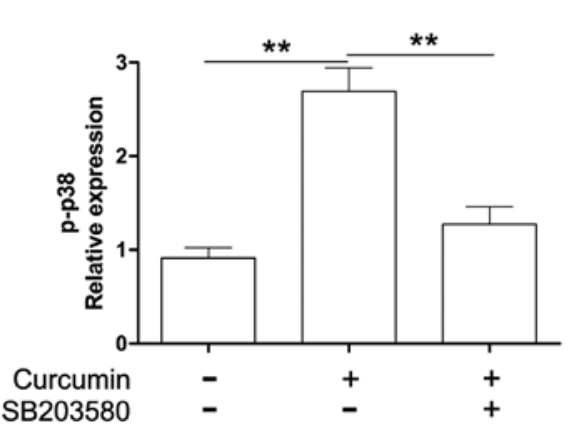

Figure 6. Effects of specific inhibitors of c-Jun N-terminal kinase (JNK) and p38 mitogen-activated protein kinase (MAPK) on curcumin-induced activation of JNK and p38 MAPK. (A and B) Y79 cells were treated in the absence or presence of $20 \mu \mathrm{M} \mathrm{JNK}$ inhibitor (SP600125) or p38 MAPK inhibitor (SB203580) for $1 \mathrm{~h}$, then treated with $80 \mu \mathrm{M}$ curcumin for $24 \mathrm{~h}$, and then the phosphorylation levels of JNK and p38 MAPK were assessed by western blot analysis. Levels of GAPDH served as a loading control. The results shown are representative of at least three independent experiments. ${ }^{* *} \mathrm{P}<0.01$. p-, phosphorylated.

or presence of $20 \mu \mathrm{M} \mathrm{JNK}$ inhibitor (SP600125) or p38 MAPK inhibitor (SB203580) for $1 \mathrm{~h}$ and then treated with $80 \mu \mathrm{M}$ curcumin for $24 \mathrm{~h}$. Protein expression was evaluated by western blot analysis. As shown in Fig. 6, SP600125 and SB203580 markedly suppressed the activation of JNK and p38 MAPK induced by curcumin. Moreover, we also found that both SP600125 and SB203580 significantly reduced curcumin-induced caspase-9/-3 activation (Fig. 7A and B) and apoptosis (Fig. 7C). These results revealed that the activation of caspase-9/-3 in the presence of curcumin may occur through the activation of JNK and p38 MAPK in Y79 cells.

\section{Discussion}

$\mathrm{RB}$ is the most common pediatric eye cancer. It is second only to uveal melanoma in terms of the frequency of occurrence of malignant intraocular tumors (24). Although chemotherapy has become an important part of the present management of 


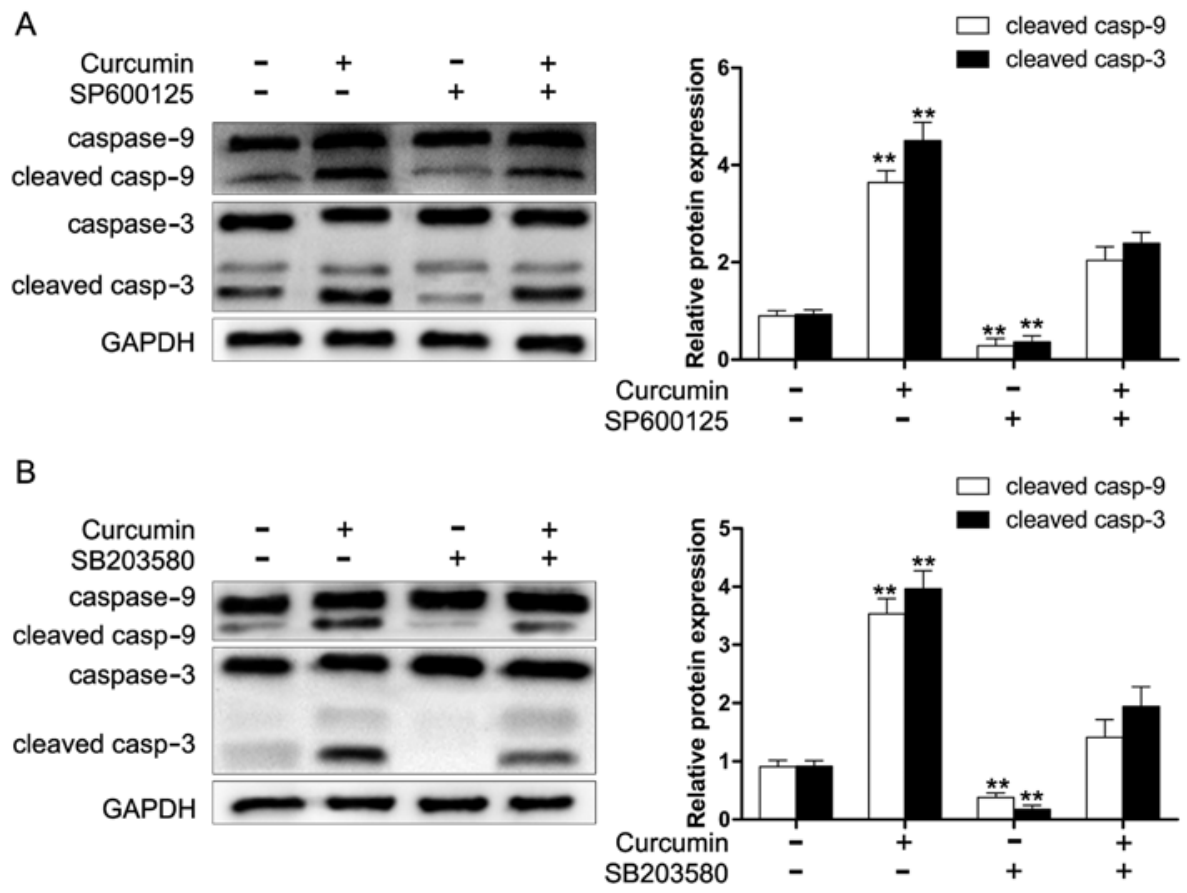

C

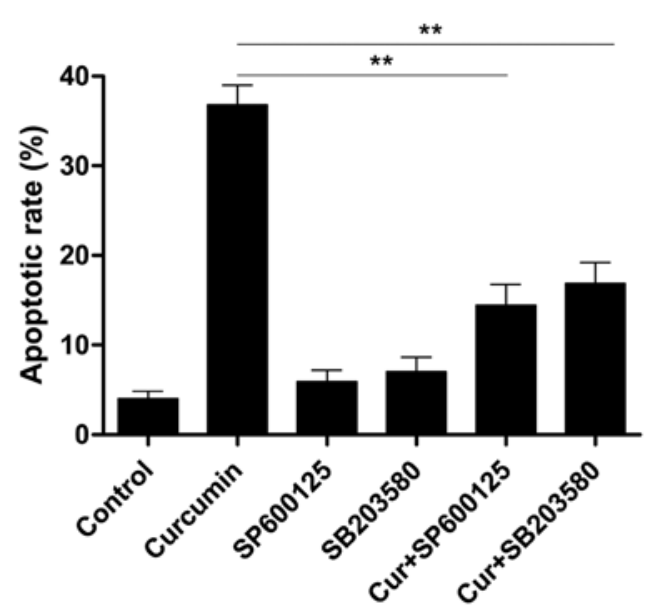

Figure 7. c-Jun N-terminal kinase (JNK) and p38 mitogen-activated protein kinase (MAPK) mediate curcumin-induced apoptosis in Y79 cells. (A and B) Y79 cells were treated in the absence or presence of $20 \mu \mathrm{M}$ SP600125 or SB203580 for $1 \mathrm{~h}$, and then treated with $80 \mu \mathrm{M}$ curcumin for $24 \mathrm{~h}$. Expression levels of caspase- 9 and -3 were determined by western blot analysis. Levels of GAPDH served as a loading control. The results shown are representative of at least three independent experiments. ${ }^{* *} \mathrm{P}<0.01$ vs. control. (C) Quantitative analysis of the total apoptotic (early and late) population. Apoptotic cells were analyzed by flow cytometry (FACS analysis) with propidium iodide (PI) and Annexin V-fluorescein isothiocyanate (FITC) staining. Data are presented as the means $\pm \mathrm{SD}$ of three independent experiments. ${ }^{* *} \mathrm{P}<0.01$. Cur, curcumin.

$\mathrm{RB}$, it causes noteworthy complications including secondary malignancies and results in long-term survival rates that remain low in developing countries $(25,26)$. Thus, the search for novel treatment modalities is imperative. Curcumin, a natural polyphenolic compound, exerts powerful growth inhibitory and apoptosis-inducing effects on cancer cells through the regulation of various signaling pathways (27-29). Although the potent anticancer effects of curcumin have been demonstrated in many types of cancer, the precise mechanism responsible for the effects of curcumin in human RB has not been fully explored. In the present study, we examined whether curcumin may potentially be used in the treatment of human $\mathrm{RB}$ and explored the potential mechanisms responsible for the anticancer effects of curcumin in Y79 cells.
Our results showed that curcumin reduced cell viability in a dose-dependent manner in Y79 cells. Cell proliferation is regulated by the cell cycle, which is a complex and stepwise process. The activity of CDKs is mediated by cyclin regulatory subunits. These form a complex with the catalytic subunit of CDKs and are controlled at a specific phase of the cell cycle $(30,31)$. In the present study, we found that curcumin treatment induced an accumulation of Y79 cells in the G1 phase of the cell cycle. We also observed that curcumin reduced the protein expression of cyclin D3, CDK2 and CDK6, and enhanced the expression of CDK inhibitor proteins $\mathrm{p} 21$ and $\mathrm{p} 27$ which suggested that changes in these protein levels appear to make a major contribution to curcumin-induced G1 arrest in Y79 cells. 
Apoptosis is a major biological process that leads to specific cell death via an intrinsic 'suicide' mechanism (32). The loss of $\Delta \Psi \mathrm{m}$ has been reported to induce cytochrome $c$ release which is essential for the activation of caspase-9 (33). A number of studies have demonstrated that caspases-9 and -3 play key roles in the apoptotic cascade $(34,35)$. Our data revealed that curcumin induced $\Delta \Psi$ m dissipation and activated the caspase-dependent apoptotic pathway in mitochondria. Moreover, the pan-caspase inhibitor ZVAD-FMK significantly reduced the curcumin-induced apoptosis of Y79 cells suggesting that activation of caspase-9/-3 is involved in the curcumin-regulated apoptosis of Y79 cells.

MAPKs consists of several subfamilies, such as ERK1/2, JNKs and p38. JNK and p38 MAPK are involved in a variety of cellular responses including cell proliferation, differentiation, and apoptosis (36-39). Our previous study also revealed that advanced glycation end products induce the apoptosis of human corneal epithelial cells through the generation of reactive oxygen species and the activation of JNK and p38 MAPK pathways (40), and it has also been demonstrated that the activation of JNK signaling mediates connective tissue growth factor expression and scar formation in corneal wound healing (41). However, the role of JNK and p38 MAPK signaling pathways in curcumin-induced apoptosis of Y79 cells was not investigated. In this study, we found that curcumin induced the activation of JNK and p38 MAPK in Y79 cells. The JNK-specific inhibitor, SP600125, and the p38 MAPK-specific inhibitor, SB203580, suppressed the activation of caspases- 9 and -3 , and inhibited the apoptosis of Y79 cells induced by curcumin. These results suggest that the activation of JNK and p38 MAPK signaling pathways plays a crucial role in curcumin-induced apoptosis of Y79 cells by regulating the activity of caspase- 9 and -3 .

In conclusion, the present study showed that curcumin exerts an antitumor effect on human RB cells by inducing cell cycle arrest and apoptosis. These findings suggest a novel therapeutic strategy for the management of RB which warrants further investigation.

\section{Acknowledgements}

The authors thank Dr Edward C. Mignot, Shandong University, for linguistic advice.

\section{References}

1. Villegas VM, Hess DJ, Wildner A, Gold AS and Murray TG: Retinoblastoma. Curr Opin Ophthalmol 24: 581-588, 2013.

2. de Moura LR, Marshall JC, Di Cesare S, Fernandes BF, Antecka E and Burnier MN: The effect of imatinib mesylate on the proliferation, invasive ability, and radiosensitivity of retinoblastoma cell lines. Eye (Lond) 27: 92-99, 2013.

3. Melamud A, Palekar R and Singh A: Retinoblastoma. Am Fam Physician 73: 1039-1044, 2006.

4. Eagle RC Jr: The pathology of ocular cancer. Eye (Lond) 27 128-136, 2013.

5. Dimaras H, Kimani K, Dimba EA, Gronsdahl P, White A, Chan HS and Gallie BL: Retinoblastoma. Lancet 379: 1436-1446, 2012.

6. Hsiao WT, Tsai MD, Jow GM, Tien LT and Lee YJ: Involvement of Smac, p53, and caspase pathways in induction of apoptosis by gossypol in human retinoblastoma cells. Mol Vis 18: 2033-2042, 2012.

7. He Y, Yue Y, Zheng X, Zhang K, Chen S and Du Z: Curcumin, inflammation, and chronic diseases: how are they linked? Molecules 20: 9183-9213, 2015.
8. Wu L, Guo L, Liang Y, Liu X, Jiang L and Wang L: Curcumin suppresses stem-like traits of lung cancer cells via inhibiting the JAK2/STAT3 signaling pathway. Oncol Rep 34: 3311-3317, 2015.

9. Jin H, Qiao F, Wang Y, Xu Y and Shang Y: Curcumin inhibits cell proliferation and induces apoptosis of human non-small cell lung cancer cells through the upregulation of miR-192-5p and suppression of PI3K/Akt signaling pathway. Oncol Rep 34: 2782-2789, 2015.

10. Yao Q, Lin M, Wang Y, Lai Y, Hu J, Fu T, Wang L, Lin S, Chen L and Guo Y: Curcumin induces the apoptosis of A549 cells via oxidative stress and MAPK signaling pathways. Int J Mol Med 36: 1118-1126, 2015.

11. Xu X, Chen D, Ye B, Zhong F and Chen G: Curcumin induces the apoptosis of non-small cell lung cancer cells through a calcium signaling pathway. Int J Mol Med 35: 1610-1616, 2015.

12. Wu H, Liu Q, Cai T, Chen YD and Wang ZF: Induction of microRNA-146a is involved in curcumin-mediated enhancement of temozolomide cytotoxicity against human glioblastoma. Mol Med Rep 12: 5461-5466, 2015.

13. Zhao Z, Li C, Xi H, Gao Y and Xu D: Curcumin induces apoptosis in pancreatic cancer cells through the induction of forkhead box $\mathrm{O} 1$ and inhibition of the PI3K/Akt pathway. Mol Med Rep 12: 5415-5422, 2015.

14. Zhu L, Han MB, Gao Y, Wang H, Dai L, Wen Y and Na LX: Curcumin triggers apoptosis via upregulation of $\mathrm{Bax} / \mathrm{Bcl}-2$ ratio and caspase activation in SW872 human adipocytes. Mol Med Rep 12: 1151-1156, 2015.

15. Yang SJ, Lee SA, Park MG, Kim JS, Yu SK, Kim CS, Kim JS, Kim SG, Oh JS, Kim HJ, et al: Induction of apoptosis by diphenyldifluoroketone in osteogenic sarcoma cells is associated with activation of caspases. Oncol Rep 31: 2286-2292, 2014.

16. Mathur A, Abd Elmageed ZY, Liu X, Kostochka ML, Zhang H, Abdel-Mageed AB and Mondal D: Subverting ER-stress towards apoptosis by nelfinavir and curcumin coexposure augments docetaxel efficacy in castration resistant prostate cancer cells. PLoS One 9: e103109, 2014.

17. Kuo JJ, Chang HH, Tsai TH and Lee TY: Curcumin ameliorates mitochondrial dysfunction associated with inhibition of gluconeogenesis in free fatty acid-mediated hepatic lipoapoptosis. Int J Mol Med 30: 643-649, 2012.

18. Hu A, Huang JJ, Jin XJ, Li JP, Tang YJ, Huang XF, Cui HJ, $\mathrm{Xu}$ WH and Sun GB: Curcumin suppresses invasiveness and vasculogenic mimicry of squamous cell carcinoma of the larynx through the inhibition of JAK-2/STAT-3 signaling pathway. Am J Cancer Res 5: 278-288, 2014.

19. Peng SF, Lee CY, Hour MJ, Tsai SC, Kuo DH, Chen FA, Shieh PC and Yang JS: Curcumin-loaded nanoparticles enhance apoptotic cell death of U2OS human osteosarcoma cells through the Akt-Bad signaling pathway. Int J Oncol 44: 238-246, 2014.

20. Zhu GH, Dai HP, Shen Q, Ji O, Zhang Q and Zhai YL: Curcumin induces apoptosis and suppresses invasion through MAPK and MMP signaling in human monocytic leukemia SHI-1 cells. Pharm Biol: July 1, 2015 (Epub ahead of print).

21. Guo Y, Zhang W, Yan YY, Ma CG, Wang X, Wang C and Zhao JL: Triterpenoid pristimerin induced HepG2 cells apoptosis through ROS-mediated mitochondrial dysfunction. J BUON 18: 477-485, 2013.

22. Shao Q, Zhao X and Yao L: Matrine inhibits the growth of retinoblastoma cells (SO-Rb50) by decreasing proliferation and inducing apoptosis in a mitochondrial pathway. Mol Biol Rep 41: 3475-3480, 2014.

23. Cao F, Liu T, Xu Y, Xu D and Feng S: Curcumin inhibits cell proliferation and promotes apoptosis in human osteoclastoma cell through MMP-9, NF- $\kappa \mathrm{B}$ and JNK signaling pathways. Int J Clin Exp Pathol 8: 6037-6045, 2015.

24. Pandey AN: Retinoblastoma: An overview. Saudi J Ophthalmol 28: 310-315, 2014.

25. Sarici A, Kizilkilic O, Celkan T and Gode S: Blue toe syndrome as a complication of intra-arterial chemotherapy for retinoblastoma. JAMA Ophthalmol 131: 801-802, 2013.

26. Suesskind D, Schrader M, Foerster MH, Ernemann U and Aisenbrey S: Cataract formation: a possible complication of intraarterial chemotherapy for retinoblastoma. Eur J Ophthalmol 24: 449-453, 2014

27. Wu J, Tang Q, Zhao S, Zheng F, Wu Y, Tang G and Hahn SS: Extracellular signal-regulated kinase signaling-mediated induction and interaction of FOXO3a and p53 contribute to the inhibition of nasopharyngeal carcinoma cell growth by curcumin. Int J Oncol 45: 95-103, 2014. 
28. Fan Z, Duan X, Cai H, Wang L, Li M, Qu J, Li W, Wang Y and Wang J: Curcumin inhibits the invasion of lung cancer cells by modulating the PKC $\alpha /$ Nox-2/ROS/ATF-2/MMP-9 signaling pathway. Oncol Rep 34: 691-698, 2015.

29. Kim HJ, Park SY, Park OJ and Kim YM: Curcumin suppresses migration and proliferation of Hep3B hepatocarcinoma cells through inhibition of the Wnt signaling pathway. Mol Med Rep 8: 282-286, 2013.

30. Sherr CJ: Cancer cell cycles. Science 274: 1672-1677, 1996.

31. Jacks T and Weinberg RA: Cell-cycle control and its watchman. Nature 381: 643-644, 1996

32. Cao A, Li Q, Y in P, Dong Y, Shi H, Wang L, Ji G, Xie J and Wu D: Curcumin induces apoptosis in human gastric carcinoma AGS cells and colon carcinoma HT-29 cells through mitochondrial dysfunction and endoplasmic reticulum stress. Apoptosis 18 : 1391-1402, 2013.

33. Gupta S, Kass GE, Szegezdi E and Joseph B: The mitochondrial death pathway: a promising therapeutic target in diseases. J Cell Mol Med 13: 1004-1033, 2009

34. Huang YC, Kuo CL, Lu KW, Lin JJ, Yang JL, Wu RS, Wu PP and Chung JG: $18 \alpha$-glycyrrhetinic acid induces apoptosis of HL-60 human leukemia cells through caspases - and mitochondria-dependent signaling pathways. Molecules 21 pii: E872, 2016.

35. Jiang YQ, Chang GL, Wang Y, Zhang DY, Cao L and Liu J: Geniposide prevents hypoxia/reoxygenation-induced apoptosis in $\mathrm{H} 9 \mathrm{c} 2$ cells: Improvement of mitochondrial dysfunction and activation of GLP-1R and the PI3K/AKT signaling pathway. Cell Physiol Biochem 39: 407-421, 2016.
36. Yiang GT, Yu YL, Lin KT, Chen JN, Chang WJ and Wei CW: Acetaminophen induces JNK/p38 signaling and activates the caspase-9-3-dependent cell death pathway in human mesenchymal stem cells. Int J Mol Med 36: 485-492, 2015.

37. Yu D, Mu S, Zhao D, Wang G, Chen Z, Ren H and Fu Q: Puerarin attenuates glucocorticoid-induced apoptosis of hFOB1.19 cells through the JNK- and Akt-mediated mitochondrial apoptotic pathways. Int J Mol Med 36: 345-354, 2015.

38. Zhen Y, Zhang W, Liu C, He J, Lu Y, Guo R, Feng J, Zhang Y and Chen J: Exogenous hydrogen sulfide promotes C6 glioma cell growth through activation of the p38 MAPK/ERK1/2-COX-2 pathways. Oncol Rep 34: 2413-2422, 2015.

39. Liu ZG, Jiao XY, Chen ZG, Feng K and Luo HH: Estrogen receptor $\beta 2$ regulates interlukin-12 receptor $\beta 2$ expression via p38 mitogen-activated protein kinase signaling and inhibits non-small-cell lung cancer proliferation and invasion. Mol Med Rep 12: 248-254, 2015.

40. Shi L, Yu X, Yang H and Wu X: Advanced glycation end products induce human corneal epithelial cells apoptosis through generation of reactive oxygen species and activation of JNK and p38 MAPK pathways. PLoS One 8: e66781, 2013.

41. Shi L, Chang Y, Yang Y, Zhang Y, Yu FS and Wu X: Activation of JNK signaling mediates connective tissue growth factor expression and scar formation in corneal wound healing. PLoS One 7: e32128, 2012. 